\title{
Plasma Surface Treatment of Powder Materials - Process and Application
}

\author{
Monika Pavlatová $^{1}$, Marta Horáková $^{2}$, Jan Hladík ${ }^{2}$, Petr Špatenka ${ }^{1}$ \\ ${ }^{1}$ SurfaceTreat, a.s., Na Lukách 669, 51101 Turnov, Czech Republic \\ ${ }^{2}$ Technical University of Liberec, Faculty of Mechanical Engineering, Department of Material Science, \\ Studentská 2, 46117 Liberec, Czech Republic
}

Correspondence to: monika.pavlatova@surface-treat.cz

\begin{abstract}
Polyolefin particles are hydrophobic, and this prevents their use for various applications. Plasma treatment is an environment-friendly polyolefin hydrophilisation method. We developed an industrial-scale plant for plasma treatment of particles as small as micrometers in diameter. Materials such as PE waxes, UHMWPE and powders for rotomolding production were tested to verify their new surface properties. We achieved significantly increased wettability of the particles, so that they are very easily dispersive in water without agglomeration, and their higher surface energy is retained even after sintering in the case of rotomolding powders.
\end{abstract}

Keywords: plasma treatment, polyolefin powders, wettability, surface energy, agglomeration.

\section{Introduction}

Cold plasma surface modification has been established as an effective and low-cost technology for modifying the surface properties of polymer materials without altering the bulk material. Modification of plastic packaging foils, plastic headlights, or automobile bumpers prior to metallization or painting are examples of industrial applications. Another application is in surface cleaning processes for removing contaminants such as grease, greasy acids, dust, and even bacteria [1].

Although powders find a range of applications in many branches of industry, e.g. painting, biotechnology, and fillings for composite materials, plasma modification of the powder surface has not been applied for modifying flat solid materials. This is due to problems connected with their three-dimensional geometry, the need for sophisticated mixing to overcome the aggregation phenomenon, and the large surface area of the powders that are to be modified.

SurfaceTreat Company has developed an original setup that enables powder hydrophilisation on an industrial scale. The aim of this paper is to present the process of powder surface modification and mainly new properties and the specific application of the modified material.

\section{Plasma technology}

Plasma discharge is a widely-used method for changing the specifications of surfaces. Plasma discharge is a simple and easy approach for changing the chemical constitution of raw materials. Basically, plasma can be generated by putting energy into a gas by heating, applying a voltage, injecting electromagnetic waves, or a combination of these methods. Due to the increasing energy, the gas particles (atoms and molecules) begin to move faster in the three spatial dimensions, and simultaneously they acquire higher rotational and vibrational energies. The particles are pulled apart due to the collisions between them, and they give rise to positively charged ions and to electrons that escape from the atoms and molecules. The principle of the plasma treatment process involves creating active particles by transporting the working gas through the plasma discharge, which changes the surface properties of the material in various ways. $[2,3]$

The bonding of new functional groups on the molecular chain during plasma surface treatment of polymer materials is one way in which this type of treatment can be used. The most frequently applied process is polymer hydrophilisation by $\mathrm{OH}$ groups. The resulting effect is a change in the surface energy, resulting in higher wettability, ability of the material to disperse, and also enhancement of the adhesion properties of the polymer to other materials.

\section{System ST 650}

SurfaceTreat Inc. SurfaceTreat developed the ST 650 pilot plant, which produces hydrophilic powder materials. The plant is based on a vacuum cham- 
ber equipped with a mechanical stirrer. The plasma is excited by two microwave power sources with total power up to $2 \mathrm{~kW}$. The development of this industrial-scale plant for powder treatment is described in detail in a previous paper [4]. Two-shift operation produces up to 3 tons per week, which is a sufficient amount to cover the delivery of material to start production, and also serves for semiindustrial tests of materials for various applications. The ST 1000 machine is now under development, and production of at least of $70 \mathrm{~kg}$ per hour is estimated for 500 micrometer particle size. The advantages of plasma treatment of powders are summarized as follows:

- surface properties can be modified without altering the bulk material

- high stability of the treatment effect

- great efficiency, together with wide variability of the process

- an environment-friendly surface modification process

Project No. FR-TI1/176 "Application of plasma modification of micro powder in industrial scale" is being implemented within the framework of the TIP programme for industrial research and development, supported by the Ministry of Industry and Trade of the Czech Republic. The project tests the modification effect on various types of powder materials, their industrial applications, and is developing an industrial-scale machine prototype in cooperation with the Technical University of Liberec, Czech Republic.

\section{Application examples}

The plasma-modified powder has already found various applications. Two characteristic industrial applications of the hydrophilized material, mainly used as an additive and/or as a raw material for rotomolding, will be described in the next section. We also participate in and support research in other applications of plasma surface treatment.

\section{Additives}

\section{- polyethylene wax}

Dispersivity is a major problem with polyolefin powders. The aim is to increase the surface energy of the particles in order to ensure dispersion without creating clusters. This problem is usually solved by using chemical dispersing agents or chemically oxidized variants for application in dispersions. Examples are polyolefin waxes, which are used as a rubresistant additive for printing inks, for modifications to surface property matting agents for paints \& coatings, for lubricants for plastics, and especially for highly demanding pigment concentrates based on PE and PP (dispersing aids).

We tested PE wax with a particle size of $\mathbf{8} \mu \mathbf{m}$. First, it was necessary to find suitable treatment conditions in laboratory equipment. The plasma treatment process is very variable in terms of pressure, working gas, and time for treatment setting. The mixing system is also very important. Then appropriate conditions were found in the production apparatus. Samples with different grades of modification were prepared and tested immediately after treatment. In this case, the wetting behavior was observed after intensive mixing (2000 rpm for 1 hour using dissolver disc $\oslash=60 \mathrm{~mm}$ ). After stopping the stirrer and waiting for $30 \mathrm{~min}$, we evaluated the settling behavior. Estimation of the wettability: 1-2 cm water phase was tolerated. All samples were compared with the commercially-produced oxidized wax (ox) benchmark system, and also with the unmodified powder zero sample. We achieved very good dispersion, comparable with or even better than the benchmark (ox), see Figure 1. The material is now being tested by the end-user. Additional testing in the form of a long-term stability test is still running, and technological adjustments are being made to the production apparatus to ensure high quality of the treatment as well as effectiveness of the process.

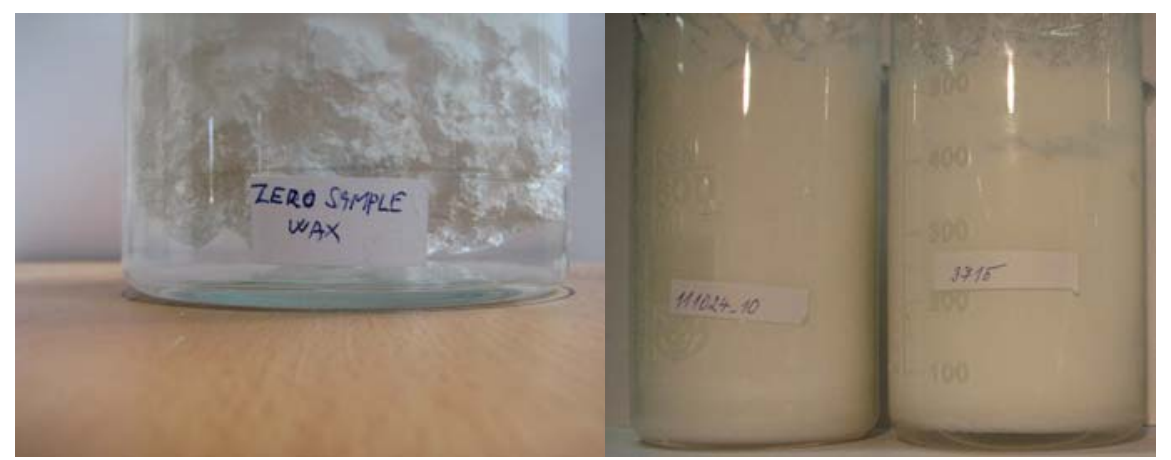

Figure 1: From the left — unmodified powder (zero sample), plasma-modified powder (111024_10), chemically oxidized powder (3715) 
- ultra-high molecular weight polyethylene (UHMWPE)

Many types of powders are classified as additives for paints, and are used, e.g., to influence the abrasion properties or the appearance. We have developed an UHMWPE treatment powder with particles $30 \mu \mathrm{m}$ in size. Very good wettability was achieved after plasma treatment, see Figure 2. The wetting behavior is observed in dispersion in distilled water to show the effect immediately after treatment. The plasma-treated powder is dispersed without any agglomerations, unlike the unmodified powder.

The wettability of the powder is then practically determined from dynamic capillarity rising measurements using a tensiometer, according to the Washburn method [5]). The suction of the material is determined as the time dependence of the weight of the penetrating benzyl alcohol at $25{ }^{\circ} \mathrm{C}$. The wettability of the powder was about $90 \%$ higher than the wettability of the unmodified sample. Various grades of treatment were tested.

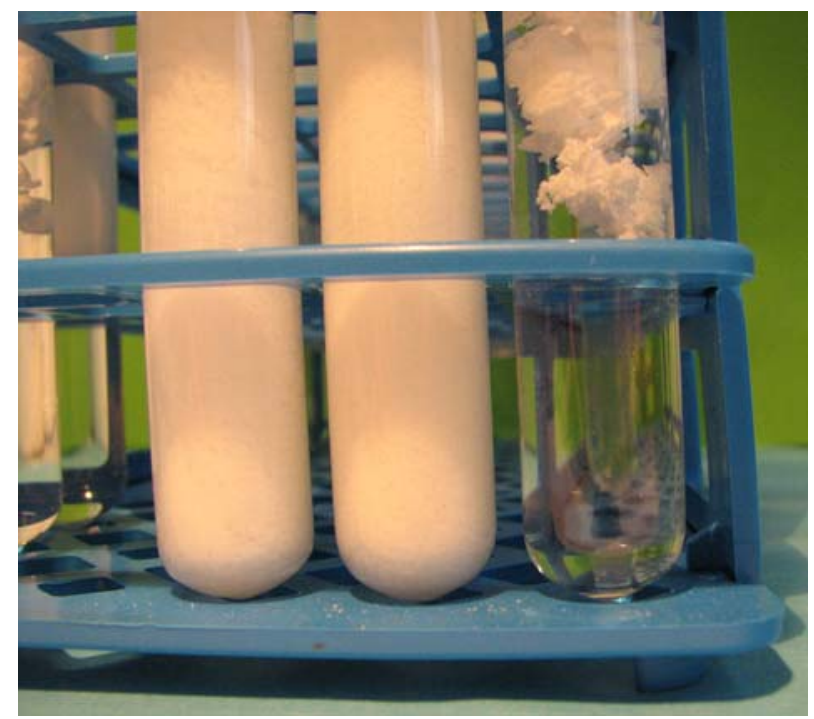

Figure 2: From the left — plasma-modified powder in 2 grades, unmodified powder

Enhancing the wettability of the powder results in effective dispersion of the powder in a liquid environment. This enables the powder to be applied as a filler in a water-based paint. ECOLOR PROFI base $\mathrm{C}$ type: 9005 was used for the test, and was then coated on a sheet metal plate as a layer of pure varnish, and as a layer with added modified powder. The modified material was immediately and very easily dispersed, without agglomeration or air bubbles. Then we tested the influence of the addition of UHMWPE to water-based paint on the abrasion resistance. A typical result of an abrasion test per- formed on the Elcometer 1720 Abrasion, Scrubbing \& Washability Tester according to norm EN ISO DIN 11998 is presented in Figures 3 and 4 . The tester was set to the fixed speed mode at 37 cycles per minute. The specimens were exposed to an abrasion tester, and the number of cycles until the paint-layer disrupts was counted. For the tests, a ratio of $10 \%$ plasma modified powder by volume was used in the varnish.

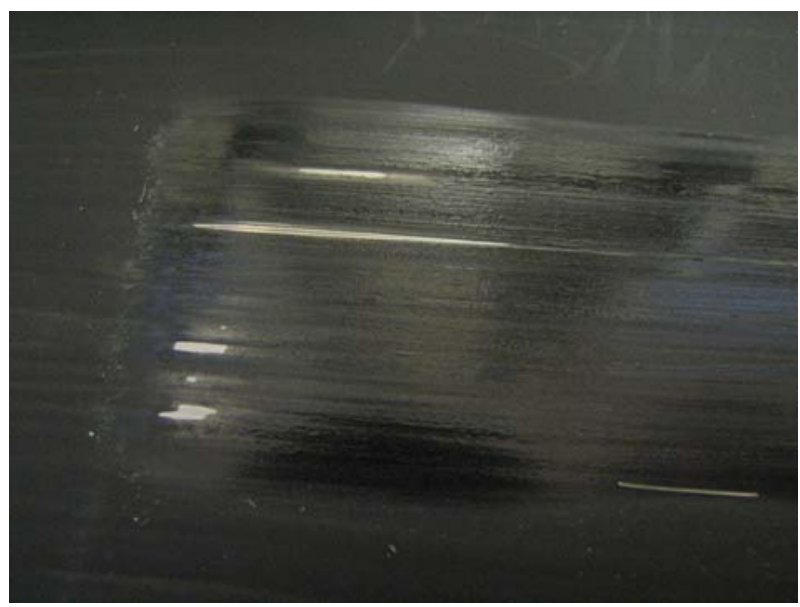

Figure 3: Pure varnish 30000 cycles, boar hair brush tool

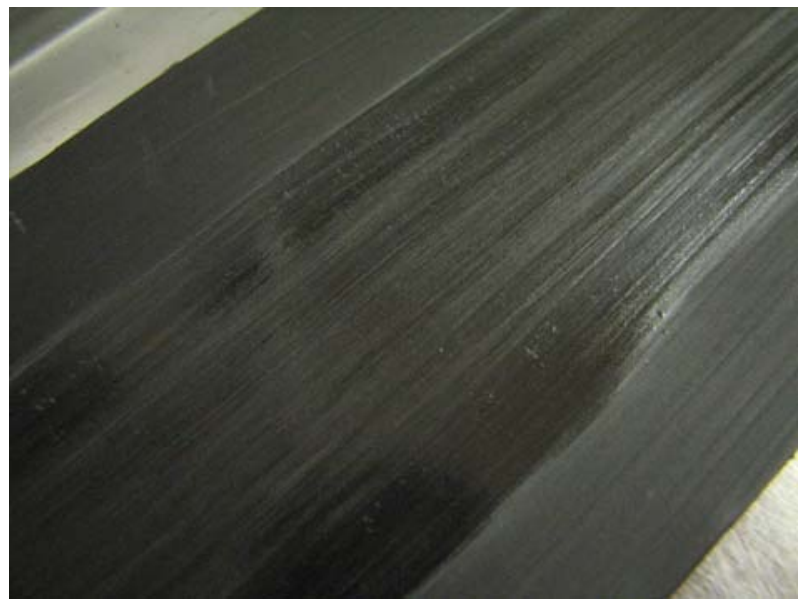

Figure 4: Varnish filled with $10 \%$ by volume plasma modified UHMWPE 135000 cycles, boar hair brush tool

The layer made of pure varnish was able to withstand 30000 cycles using the boar hair brush tool before the first wear through occurred, see Figure 3. The layer of varnish with $10 \%$ by volume plasma modified powder was able to withstand 135000 cycles using the hair brush tool without any wear through, see Figure 4. 
Adding unmodified PE into the paint also raised the abrasion resistance of the coating, but there were significant problems with admixing the unmodified filler into the paint. The paint layer with plasma modified powder was smooth and homogeneous. We are working in cooperation with lacquer and paint producers, and various plasma-modified materials are being tested by customers, using certified laboratories.

\section{Powders for rotomolding}

Plasma surface treatment of solids is currently more widely employed. It can replace chemical wet processes or flame interference, which are used to enhance adhesion properties.

We are able to go even further, and to treat in powder form and then sinter a product with new surface properties using a rotomolding technique. The surface is directly prepared for painting, gluing, printing or foaming without any additional pretreatment. The characteristics of the material, i.e. impact strength, ESCR and other major specifications, are the same as for traditional materials, and the processing also remains unchanged [6]. The enhanced surface hydrophility remains, even after sintering.
We tested various types of PE powders suitable for rotomolding. The effect of treatment was verified by measuring the surface energy after sintering using test inks and test pens by ARCOTEST GmbH.

Surface energy is a decisive criterion for the adhesion of printing ink, glue, varnish, etc., to many plastic and metal surfaces. It is given in $\mathrm{mN} / \mathrm{m}$. A value of $38 \mathrm{mN} / \mathrm{m}$ is often mentioned as a general limit. If the surface energy level is below this value, the adhesion is poor; above this value, the adhesion is good or sufficient [7]. Our samples sintered from modified powder have surface energy values about $10 \mathrm{mN} / \mathrm{m}$ higher than the zero (unmodified) samples, always depending on the type of material and the modification grade, see Table 1 . The particle size of the tested materials was approximately $500 \mu \mathrm{m}$.

All materials mentioned here reached or exceeded the required limit value of $38 \mathrm{mN} / \mathrm{m}$.

As practical tests for industrial applications, we tested the adhesion of the water-based paint layer on a surface sintered from the modified material without any additional pre-treatment of the surface. Then an adhesion test was performed using the cross cut method, according to ISO 2409. The plasmamodified material samples produced very good results. The sample sintered from unmodified powder received classification No 5 (Figure 5), and the sample sintered from plasma-modified powder received classification No 0 or 1 for (Figure 6).

Table 1: Overview of the surface energy measured on a surface sintered from unmodified and modified powder for different types of material

\begin{tabular}{|l|c|c|c|c|c|}
\hline Material/Producer & Type & $\begin{array}{c}\text { Density } \\
{\left[\mathrm{g} / \mathrm{cm}^{3}\right]}\end{array}$ & $\begin{array}{c}\text { MFI } \\
{[\mathrm{g} / 10 \mathrm{~min}]}\end{array}$ & $\begin{array}{c}\text { Surface energy } \\
{[\mathrm{mN} / \mathrm{m}]} \\
\text { before treatment } \\
\text { ARCOTEST } \\
\text { pens/inks })\end{array}$ & $\begin{array}{c}\text { Surface energy } \\
{[\mathrm{mN} / \mathrm{m}]} \\
\text { after treatment } \\
(\text { ARCOTEST } \\
\text { pens/inks })\end{array}$ \\
\hline $\begin{array}{l}\text { Borecene RM 8343 } \\
\text { Borealis }\end{array}$ & LMDPE & 0.9340 & 6.0 & $30 /<35$ & $44 / 41-44$ \\
\hline $\begin{array}{l}\text { mPE M 3581 UV S01 } \\
\text { Total Petrochemical }\end{array}$ & MDPE & 0.9350 & 6.0 & $30 /<35$ & 44 \\
\hline $\begin{array}{l}\text { Lupolen 3621 M RM } \\
\text { LyondellBasell }\end{array}$ & MDPE & 0.9355 & 7.5 & $30 /$ n.a. & $42-44 / 41-44$ \\
\hline $\begin{array}{l}\text { Surpass RMS 244 UG } \\
\text { Nova Chemicals }\end{array}$ & HDPE & 0.9440 & 1.7 & $30-36 /<35$ & $42-44 / 41$ \\
\hline $\begin{array}{l}\text { DOWLEX 2631.10UE } \\
\text { Dow Chemical Comp. }\end{array}$ & PE & 0.9350 & 7.0 & $30 / 28$ & $38-40 / 38-41$ \\
\hline $\begin{array}{l}\text { ICORENE 3590 ICO } \\
\text { Polymers }\end{array}$ & LMDPE & 0.9350 & 9.0 & $30 / 28$ & $38 / 44$ \\
\hline
\end{tabular}




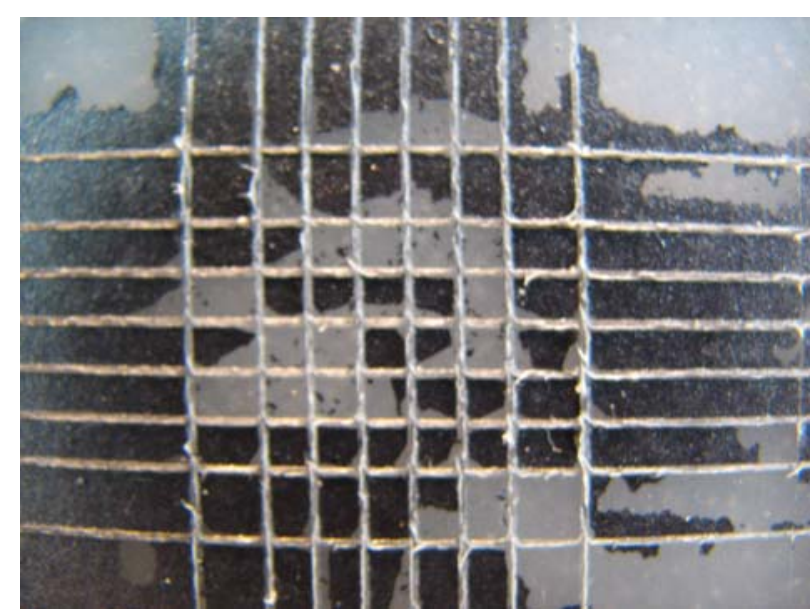

Figure 5: CROSS CUT TEST (WATER-based paint) on the surface sintered from unmodified PE powder

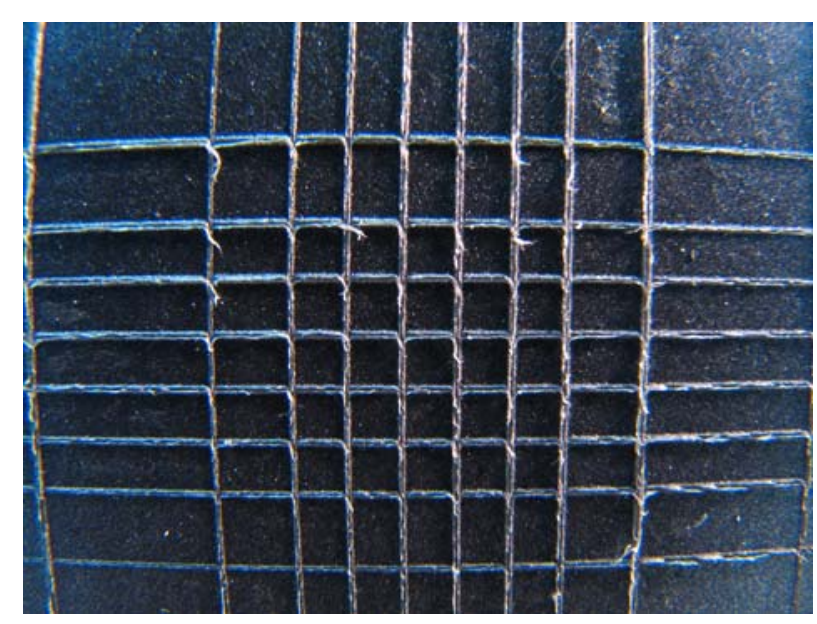

Figure 6: Cross cut test (water-based paint) on the surface sintered from plasma-modified PE powder

The adhesive bonding ability of the sintered parts was tested on the adhesion of PUR foam to a surface sintered from plasma-modified powder. The specimen was created in the form of sandwich, and consisted of one layer of sintered plasma-modified PE material, a layer of standard PUR foam, and another layer of sintered plasma-modified material. Other materials were also tested. It is known that there is no adhesion between PE and PUR. In our case, we achieved very good results using only a manual strength test. In this test, the adhesion between PE and PUR foam was higher than the cohesion of the foam itself.

Adhesion between sintered plasma-modified PE and PUR foam parts could be used in the case of foam plastic products, such as seats or thermo boxes. There is also significant enhancement of the entire body strength, which facilitates construction. At the same time, the so-called multilayer rotomolding process seems to be a very interesting area for applying plasma-modified powder. For example, fuel tanks could be produced using this process, where the plasma-modified powder can act as an adhesive, e.g. for an inner barrier layer.

\section{Conclusion}

This paper has presented a plasma treatment method for powder materials. Practical examples have shown their industrial applications. Treated polyolefin has been applied as a filler in water-based suspensions, significantly enhancing the wear resistance of the coating. Parts with hydrophilic surfaces can be produced by applying the raw material for producing parts using rotomolding technology. These parts can be directly painted or glued, without any pretreatment.

Plasma treatment of powder on an industrial scale has been developed. The ST-650 pilot plant has a production capacity of up to 3 tons per month, which is sufficient for small-series production, and provides material for industrial testing.

Further development is in progress. Plasma treatment of nano-powders will open up broad new areas of application.

\section{Acknowledgement}

This work has been partially supported by the Ministry of Industry and Trade of the Czech Republic; project No. FR-TI1/176.

\section{References}

[1] Lebert, R., Neff, W., Pochner, K.: Atmospheric Pressure Gas Discharges for Surface Treatment, Surface and Coating Technology, 74-75, 1995, 394-398.

[2] Abdul-Kader, A. M., Kelany, A. M., Radwan, R. M., Turos, A.: Surface free energy of ultra-high molecular weight polyethylene modified by electron and gamma irradiation, $A p$ plied Surface Science, Vol. 255, Issue 17, 2009, p. $7786-7790$.

[3] Fellenberg, R., Kickuth, R., Reichel, K.: Plasma Technology Process Diversity + Sustainability, German Federal Ministry of Education and Research, Printec Offset, Kassel, 2001, p. 6-12.

[4] Hladík, J., Peciar, M., Špatenka, P., Zítko, M.: Plasma Treatment of Micropowder - From Laboratory Experiments to Production Plant, 52nd Annual Technical conference Proceedings of the Society of Vacuum Coaters, 2009, p. 537-540. 
[5] Washburn, E. W.: Phys. Rev. Ser. 2, 17, 1921, 273.

[6] Boersch, D. E., Knoth, P., Pfitzmann, A.: Plasma modified polyolefin powders for rotational molding, Proceedings: 61st SPE Annual Technical Conference (ANTEC), vol. 1 of Processing Ses- sion T13 - Material Formulation and Properties, p. 1278-1281, Nashville, May 4-8 2003. Society of Plastics Engineers.

[7] ARCOTEST Manual - Test Inks for testing the surface energy. 